\title{
Itch in diabetes: a common underestimated problem
}

\author{
Aleksandra A. Stefaniak, Iwona Chlebicka, Jacek C. Szepietowski \\ Department of Dermatology, Venereology and Allergology, Wroclaw Medical University, Wroclaw, Poland \\ Adv Dermatol Allergol 2021; XXXVIII (2): 177-183 \\ DOI: https://doi.org/10.5114/ada.2019.89712
}

\begin{abstract}
Introduction: Diabetes mellitus is associated with several skin manifestations, however the association with itch remains unclear.

Aim: To present a detailed literature review in order to analyse the frequency, pathogenesis, and course of itch in diabetes mellitus.

Material and methods: Data were collected from the PubMed and Cochrane databases. Articles were excluded if the populations presented with comorbidities or received treatment with drugs affecting the skin. Also, animal studies, studies with poor methodology and pilot studies were excluded.

Results: Among the 5 original articles included, the epidemiology of itch in diabetes varies from $18.4 \%$ to $27.5 \%$. Two main factors are associated with the pathogenesis of itch in diabetes, namely skin xerosis and diabetic polyneuropathy. It is still poorly defined how glycaemic control is associated with generalized itch. No treatment of choice is available; however, topical therapy (emollients) provides significant relief in varying percentages of patients. Conclusions: The results indicate a benefit of diabetes screening in individuals presenting with chronic itch without primary skin lesions.
\end{abstract}

Key words: diabetes mellitus, itch, pruritus.

\section{Introduction}

Diabetes mellitus (DM) and its complications are a major problem worldwide. The International Diabetes Federation estimated that 1 in 11 adults aged 20-79 years (415 million adults) had diabetes mellitus globally in 2015 [1]. DM is a problem of growing importance, this estimation is projected to rise to 642 million by 2040, and the largest increases will come from the regions experiencing economic transitions from low-income to middle-income levels [1]. The reasons for the escalating epidemic of DM are multiple, including population aging, economic development, urbanization, unhealthy eating habits, and sedentary lifestyles. DM is a group of metabolic disorders characterized by high blood glucose levels due to defective secretion and/or action of insulin [1]. Symptoms suggesting the presence of diabetes include polyuria; increased thirst and weight loss that cannot be explained by intended weight reduction. There are also other, less typical symptoms and signs such as fatigue and somnolence, purulent skin lesions, and inflammatory conditions of the genitourinary tract. In the case of the onset of symptoms, a random venous plasma glucose level should be measured.
According to WHO, DM is divided etiologically to the following main groups:

1) Diabetes type 1, Insulin Dependent Diabetes Mellitus (IDDM),

2) Diabetes type 2 (DM2),

3) Other specific forms of diabetes and

4) Gestational diabetes [2].

Over $95 \%$ of diabetes mellitus cases are DM2 and IDDM [3].

Chronic hyperglycaemia is associated with damage, dysfunction, and failure of various organs, in particular eyes, kidneys, nerves, heart, and blood vessels [4]. Skin disorders are present in $79.2 \%$ of patients with DM, and cutaneous disease may appear as the first sign of DM or develop at any time in the course of the disease. Noninfectious and infectious diseases have been described as dermatologic manifestations of diabetes mellitus [5-7]. In diabetes, underlying pathophysiology, the course of the disease, coexisting comorbidities and medications - all tend to predispose patients to develop itch.

Itch is defined as an unpleasant sensation that leads to intensive scratching [8]. It is a common and distressing symptom occurring not only in dermatological conditions but also in chronic systemic diseases [8, 9], posing a high

Address for correspondence: Aleksandra A. Stefaniak MD, Department of Dermatology, Venereology and Allergology, Wroclaw Medical University, 1 Chałubińskiego St, 50-368 Wroclaw, Poland, e-mail: aleksandra.stefaniak@student.umed.wroc.pl Received: 29.08.2019, accepted: 18.09.2019. 
burden and decrease in quality of life (QoL) of an affected individual $[10,11]$. According to the International Forum for the Study of Itch (IFSI), the etiologic classification of chronic itch comprises 6 categories: (I) dermatologic, (II) systemic, (III) neurologic, (IV) psychogenic/psychosomatic, (V) mixed, and (VI) others [12]. Most researchers classify itch in diabetes as systemic itch [13], however some authors suggest mixed aetiology of diabetic itch with the additional involvement of both dermatologic and neurologic components [14-16]. Diabetes mellitus appears in most lists of the causes of generalized itch but is not common. Localized itch, for example pruritus vulvae, is common and often associated with candidal infection [17]. Only a few studies investigated the occurrence of itch in diabetes; however, these research projects used inconsistent definitions and different tools for itch evaluation and included heterogeneous diabetic populations [18].

Here, we present a detailed literature review in order to analyse the frequency, pathogenesis, and the course of itch in diabetes mellitus. Moreover, basing on the literature review, the treatment modalities for diabetic itch have been proposed.

\section{Methodology}

The search was conducted following the PRISMA guidelines [19]. The final search date was 21 August 2019. Terms included in the search, adjusted to the different databases were: "(pruritus OR itching) AND (diabetes mellitus OR diabetes OR diabetic OR diabetics OR non-insulin dependent diabetes mellitus OR Type 2 diabetes mellitus OR Type 1 diabetes mellitus OR insulin dependent diabetes OR insulin resistance OR impaired carbohydrate metabolism OR hyperinsulinaemia)". Additionally, for each review part, additional search terms (epidemiology/pathophysiology/treatment) were used.

We excluded studies of populations with confounding conditions like malignancies, thyroiditis, gestational diabetes and treatment with drugs affecting the skin. Moreover, we excluded animal studies, studies with poor methodology and pilot studies. Initially we also did not include studies focusing on more than one cutaneous manifestation, however, because only very few original papers related to the topic were found, we decided to take those studies into consideration as well. In some of the oldest articles, both the full text and the abstract were missing. These were not included in the final analysis. The included articles were screened for relevant cross-references. The selection process resulted in a total of 5 articles focusing precisely on itch in diabetes and all together 28 original papers focusing on cutaneous changes in diabetes including itching. Some of the articles focused on more aspects of itch in diabetes, so they were included into different parts of the analysis. Details of the selection process are presented in Figure 1.

\section{Epidemiology}

Epidemiologic aspects of itch in diabetes vary according to data presented in the literature. Only a few studies focused on itch investigation in diabetes. While prior studies [20, 21] showed no discernible relationship, recent papers from Taiwan and Japan [16, 22] demonstrated a correlation between diabetes and itch.

At the beginning of the 1980s, Kantor and Lookingbill [20] described a group of 44 patients with generalized itch; in this group $9.09 \%$ of patients suffered from DM.

Neilly et al. [21] in 1986 assessed 300 DM and 100 non-DM hospital outpatients for the presence of generalized and localized itch. Pruritus vulvae was significantly more common in diabetic women (18.4\%) than in controls (5.6\%) and was significantly associated with poor diabetes control (mean glycosylated haemoglobin level lower than 12\%). Generalized itch occurred in 8 diabetic patients only (2.7\%) and was not significantly more common than in nondiabetic patients. Ko et al. [22] in 2013 analysed the data of 385 DM patients participating in the diabetes care program in Taiwan. Generalized itch was noted in $27.5 \%$ of the patients.

Patients classified to the group of DM type 2 with a higher postprandial glucose level had a higher probability of generalized itch $(\mathrm{OR}=1.41,95 \% \mathrm{Cl}$ : 1.05-1.90, $p=0.02$ ).

A large multicentre study in Japan conducted by Yamaoka et al. [16] of 2,656 diabetic outpatients and 499 nondiabetic subjects revealed that the prevalence of truncal itch of unknown origin (TPUO) in diabetic subjects was significantly higher than in age-matched nondiabetic subjects (11.3 vs. $2.9 \%, p=0.0001$ ). The prevalence of total itch in diabetic subjects was significantly higher than in nondiabetic subjects ( 26.3 vs. $14.6 \%, p<0.001$ ). Interestingly, the prevalence of other forms of itch was not different between the two analysed groups. Table 1 shows details of the relevant epidemiological studies specifically dealing with itch in DM.

The other research projects were mostly examining cutaneous manifestations of diabetes, including itch. Historically, in late 1920s, Greenwood [23] was the first to show interests in itch in DM in his study of 500 DM cases. He showed that $6.6 \%$ of patients examined either had itch or had had it in the past. Out of these cases, 3.2\% had generalized and 3.4\% localized itch.

In a letter to the editor published in JAMA, Scribner [24] in the 1970s, attributes generalized itch and itch of the scalp to poorly controlled diabetes mellitus, but mostly he relied on his medical practice and general beliefs. However, a study done by Valdes-Rodriguez et al. [25] in 2015 on elderly patients, found that in the diabetic group, the presence of the disease correlated with scalp itch. In patients with DM2 $(n=93), 40 \%$ experienced itch on the scalp, in contrast with $17.5 \%$ of patients without DM ( $p=0.037)$. Tseng et al. [15] who also studied the 


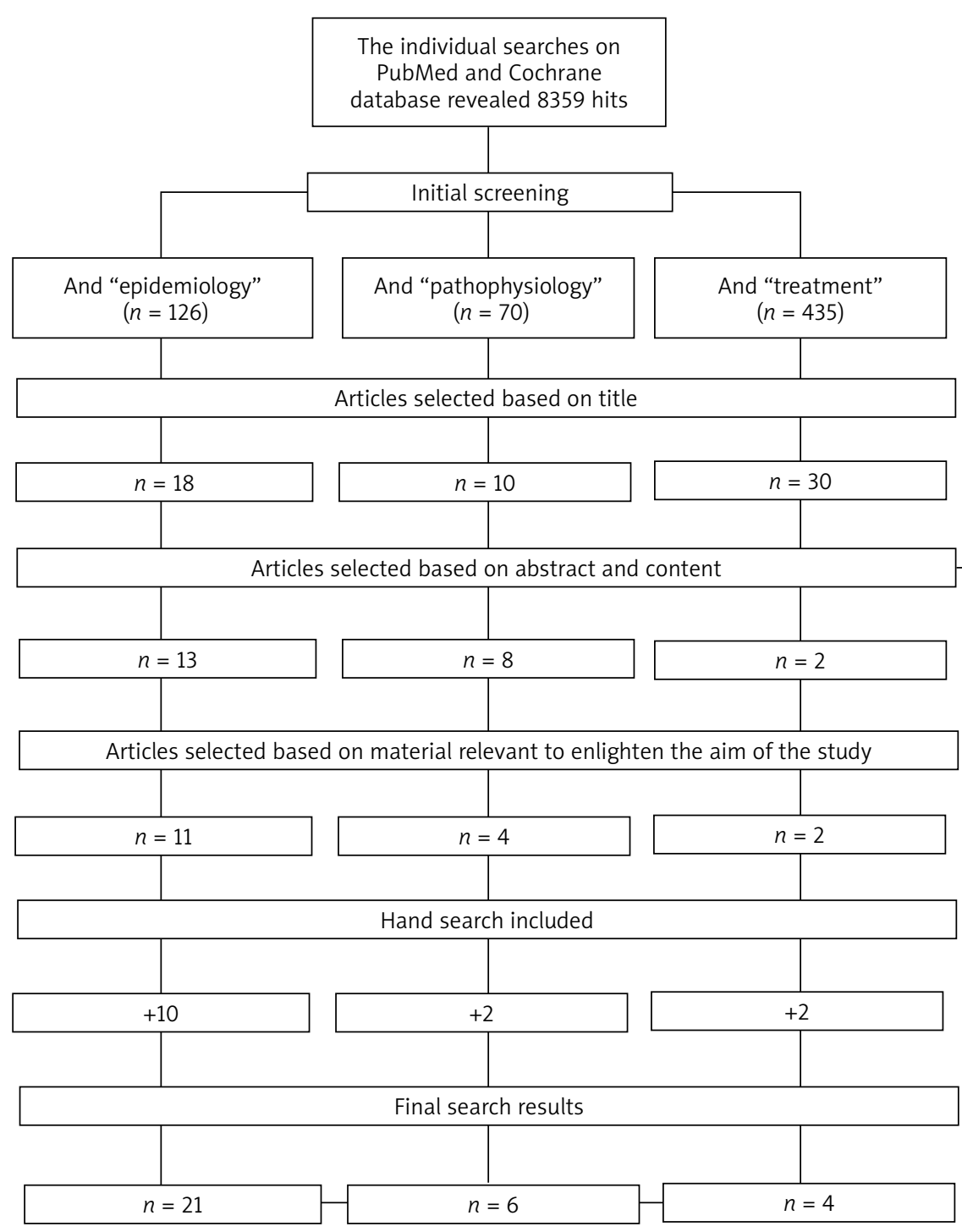

Exclusion criteria: published language other than English, studies based on animals, pilot studies, reviews, cases, comorbidities, drugs affecting skin or poor methodology

*The amount of hits contains several duplicates.

Figure 1. Literature selection process according to PRISMA guidelines

elderly population, however, male-only, also claimed that itch was significantly associated with $\mathrm{DM}(\mathrm{AOR}=12.86$, 95\% Cl: 4.40-37.59; $p<0.001)$. In another study based on female geriatric population [26] $(n=36)$, generalized itching $(20 \%)$ and pruritus vulvae (33.3\%) were commonly presenting complaints. In Poland [27], in a study conducted on 198 elderly subjects, no correlation between itch and diabetes mellitus has been found. In 1985, Hillson et al. [28] checked 235 newly diagnosed and untreated patients with DM2 and $20 \%$ of them had itch.

In the following studies, itch was reported in $4.5 \%$ of patients $(n=200)$ [29], 9.9\% $(n=106)$ [30], 10\% $(n=$ 100) [31] and $13.33 \%(n=60)$ [32]. In a study comprising 350 individuals, by Ahmed et al. [33], itch was reported in $7.1 \%$. Another perspective was chosen by Alizadeh et al. [13] who examined 5127 patients referred to the dermatologic clinic with chronic generalized itch without primary skin lesions $-12.5 \%$ of them had DM.

During the same time, among Egyptian diabetic patients [34], 11\% had itch $(n=100)$. However, in this study, only patients with at least one skin manifestation of DM were selected. The same criteria were applied in the Indian study [31] in which the incidence was 30\%, and also in the study done by Al-Mutairi et al. [35] in Kuwait. Al-Mutairi et al. [35] in their study showed the highest known incidence of itch - nearly half (49\%) of the examined patients complained of the symptom.

Anogenital pruritus, commonly known in the case of women as pruritus vulvae, was also a subject of studies during the years. A large randomized controlled trial in Denmark by Drivsholm et al. [36] including patients aged $\geq 40$ years with newly diagnosed DM2 $(n=1,543)$ re- 
Table 1. Prevalence of itch in diabetes mellitus

\begin{tabular}{lcccc}
\hline Reference & Study design & Participants, $n$ & Patients affected by itch & Prevalence of itch \\
\hline Neilly et al., 1986 [21] & Case-control & $\begin{array}{c}300 \text { patients, } \\
100 \text { controls }\end{array}$ & $18.4 \%$ & Point prevalence \\
\hline Yamaoka et al., 2010 [16] & Cross-sectional & $\begin{array}{c}\text { 2,656 outpatients, } \\
499 \text { inpatients }\end{array}$ & $26.3 \%$ & No data \\
\hline Ko et al., 2013 [22] & Cross-sectional & 385 patients & $27.5 \%$ & $\begin{array}{c}\text { Prevalence after the } \\
\text { diagnosis of diabetes }\end{array}$ \\
\hline
\end{tabular}

vealed a prevalence of $27.2 \%$ of genital itching. In a study [37] comprising 100 individuals, localized anogenital itch was present in $19 \%$ of patients.

There are even fewer studies focusing on diabetes type 1. In Iran, Farshchian et al. [18] in their study examined patients for cutaneous manifestations of DM $(n=155) .17 .4 \%$ of patients with IDDM had suffered from significant itching, comparing to $27 \%$ of patients with DM2. In Israel [38], 2\% of IDDM $(n=238)$ patients were affected by itching sensations.

Summarizing, taking into account epidemiological data concerning itch in DM, one may draw a conclusion that there is a limited number of studies dealing specifically with itch in this group of patients. Most studies lack the information whether the point prevalence, life-time prevalence or incidence of itch was noted. It seems that $18.4 \%$ to $27.5 \%$ of patients with DM2 may suffer from itching. Studies on itch in IDDM, employing currently accepted methodology, are practically missing. Studies on itch prevalence, based on all clinical cutaneous manifestations analysed in DM patients, have given conflicting results and are difficult to be compared with each other due to non-homogenous methodology.

\section{Pathogenesis}

Pathogenesis of pruritus in DM is not fully understood and various factors are described as contributing to the development of this symptom. Currently, the researchers believe that there are two main factors associated with the itch in diabetes, namely skin xerosis and diabetic polyneuropathy.

\section{Skin xerosis}

It is well known that generalized itching may occur in clinically inconspicuous dry skin [16, 22]. Seité et al. [39] assessed 40 diabetic patients with regard to stratum corneum (SC) hydration (measured by a corneometer), the skin barrier function (transepidermal water loss - TEWL) (using a tewameter), skin elasticity (using a cutometer) and itching intensity. Emollient application induced a significant increase in skin hydration associated with a remarkable reduction in TEWL, and - consequently alleviated itching.
Diabetes impairs wound healing [40-42]. Diabetes induces an increase in advanced glycosylation products in the collagen of the dermis. This glycosylation also favours oxidation, and it may be responsible for some of the skin changes associated with aging and this process is accelerated in persons with higher blood sugar levels [43, 44]. The significance of insulin as an essential growth factor for cultured keratinocytes and the substantial influence of insulin on keratinocyte proliferation [45], migration $[46,47]$ and differentiation [48] implies that the distorted keratinocyte phenotypes in patients with diabetes are an important factor in their impaired wound healing. Moreover, it is reasonable to presume that the abnormal proliferation and differentiation of keratinocytes in the epidermis of DM patients change the functions of the SC. Stratum corneum prevents excessive water loss and penetration of pathogens and allergens. All of the above mentioned factors may contribute to the pathogenesis of itching in DM. It was shown that diabetes influences the epidermal barrier quality. Sakai et al. $[49,50]$ put forward a hypothesis that patients with DM tend to develop a reduced hydration state of the SC together with decreased sebaceous gland activity. However, Seirafi et al. [51], in a case-control study, demonstrated no significant difference in SC hydration and TEWL in DM patients compared with age- and gender-matched healthy people. They additionally pointed to a lower acoustic wave propagation speed (related to skin elasticity) in DM subjects.

\section{Neuropathy}

Newer theories have suggested that itch in DM may be associated with polyneuropathy. Diabetes mellitus is the most common cause of small-fibre polyneuropathy in developed countries [52]. One of the theories suggested that diabetic polyneuropathy with correlated dysfunction of sweating due to impairment of the sympathetic nervous system may play a role in the pathogenesis of itch in DM [53].

Fluctuations in glucose levels seem to be the reason for dry skin in diabetes, however autonomic neuropathy may also play a significant role in skin dehydration in diabetic patients.

As a result of small-fibre polyneuropathy, diabetic patients can develop a neuropathic itch. A recent study in Ja- 
pan [16] has mentioned truncal itch as a frequent clinical manifestation of neuropathy in diabetic patients. Multiple logistic regression analysis revealed that numbness of soles and palms and lack the Achilles tendon reflex (ATR) were independent risk factors for itch in age, sex, duration of diabetes, and glycolized haemoglobin $\left(\mathrm{HbA}_{1 \mathrm{c}}\right)$ levels. Autonomic nerve function was tested using the head-up tilt test and heart rate variability test. A fall of systolic blood pressure in diabetic subjects with itch was significantly impaired compared with that in subjects without it. In the patients with diabetic polyneuropathy judged by bilateral ATR areflexia, the prevalence of truncal pruritus of unknown origin was $20.5 \%$ compared to asymptomatic patients with polyneuropathy where itch was observed in $15.2 \%$.

\section{Other factors}

Only a few studies focused on the itch and a blood glucose level. The limitations of these studies are the usage of different parameters, such as the fasting plasma glucose, postprandial glucose, and $\mathrm{HbA}_{1 c}$ levels. The fasting plasma glucose (FPG) shows the hyperglycaemic state at the time of the measurements, while the $\mathrm{HbA}_{1}$ reflects the average blood glucose levels in the past 7-8 weeks preceding the measurements. It seems that there is a correlation between postprandial glucose and generalized itch in patients with DM [22]. Ko et al. [22] found out that patients with a higher postprandial glucose level had a higher probability of generalized itch $(p=0.02)$. Hillson et al. [28] found out a correlation between the fasting plasma glucose and generalized pruritus in newly diagnosed and untreated patients with DM2.

When it comes to $\mathrm{HbA}_{1 \mathrm{c}}$ levels, different studies showed different results. In both studies mentioned above, no relationship between $\mathrm{HbA}_{1 c}$ levels and generalized itching has been found [21, 22]. However, in a study designed by Afsar and Elsurer [54] on 75 patients (diabetic/nondiabetic, 29/46), the intensity of itching assessed with Visual Analog Scale (VAS) was higher in diabetic patients compared with nondiabetic patients ( $4.7 \pm 2.8$ vs. $3.0 \pm 1.0, p=0.015$ ). Additionally, in diabetics, VAS itch score was independently related to $\mathrm{HbA}_{1 \mathrm{c}}(\beta=+0.310$, $p=0.027$ ). It is still poorly defined how glycaemic control is associated with generalized itch.

Unfortunately, most of the studies lack data showing whether the itch was followed by bacterial or fungal infections. In the study of Al-Mutairi et al. [35], diabetic patients hospitalized in the dermatologic ward were assessed for the presence of itch. Itch was one of the most common cutaneous signs of diabetes in $49 \%$ of $(n=106)$ patients, followed by fungal (78.8\%) and bacterial (51.9\%) infections.

\section{Treatment}

Only a few studies focused on treatment of itch in diabetes and most of them assessed topical therapy.
Seité et al. [39] performed a study on DM patients $(n=40)$, who were treated with the emollient (containing $5 \%$ urea associated with $0.2 \%$ hydroxyethylpiperazine ethane sulfonic acid) twice daily for 1 month on one arm and one leg, in normal conditions. The itch was also evaluated by 100-mm VAS starting from $2.0 \pm 2.91$ at baseline, whereas on day 30 a marked reduction was noted (0.10 $\pm 0.5, p<0.0001)$. A significant improvement was also observed in skin hydration associated with a significant reduction in the desquamation index and TEWL.

There is a lack of research about the potential role of emollients in skin dryness prophylaxis in diabetes, as well. Also, the problem of dry skin in diabetes is often neglected by general practitioners. Narbutt et al. [55] proposed the application of an emollient with benfotiamine and Biolin prebiotic as prophylaxis of skin dryness and itch in skin care of DM patients, however there were no data supporting itch alleviation.

If emollients are contraindicated or less well tolerated, Ibrahim et al. [56] stated that topical treatment with clove oil may be also effective in chronic itch of systemic origin. He assessed topical clove oil use in 10 diabetic patients, comparing to 9 patients with diabetes treated with topical petrolatum as placebo for 2 weeks. Itch was assessed using 5-D itch scale. All the diabetic patients treated with topical clove oil showed a significant improvement in itch alleviation $(p=0.02)$ and comparing to the placebo group.

Diabetic neuropathy is often associated with oxidative stress. Therefore, an attempt was made to treat this disease with an antioxidant, lipoic acid, in Korean diabetic patients with distal symmetric polyneuropathy (DSP) [57]. Lipoic acid was administered orally using $600 \mathrm{mg}$ once daily for 8 weeks to 61 diabetic patients with symptomatic polyneuropathy. Neuropathic symptoms (pain, burning sensation, paraesthesia, and numbness) were scored at baseline as well as at 4 and 8 weeks following treatment. Efficacy was evaluated in 38 patients who had completed the study according to the protocol. All the individual scores for neuropathic symptoms (pain, burning sensation, paraesthesia, and numbness) were also significantly reduced at 4 weeks and further decreased at 8 weeks $(p<0.05)$. As itch is commonly associated with the burning sensation and described by patients as numbness, lipoic acid seems to be a promising medication to alleviate neuropathic itch in patients with DM.

There is a lack of research on other treatment options for patients with itch and diabetes. However, basing on the treatment options for non-dermatological itch and other skin complications of diabetes, the treatment modalities for diabetic patients may be proposed.

Itch management begins with applying preventive measures to avoid factors that foster dryness of the skin, such as e.g. dry climate, heat (e.g. sauna), ice packs, frequent washing and bathing and contact with irritant substances [10]. It is recommended to use mild, non-alkaline 
cleansers and to bath in lukewarm water for less than 20 min. Wearing loose-fitting, soft clothing permeable to the air, like cotton, is also beneficial. Patients may also appreciate wet, cooling or fat-moist-wrappings, black tea wrappings or relaxation techniques prescribed by the doctor.

The lifestyle changes, highly recommended by the American Diabetic Association, are mostly the use of emollients in the treatment of itch as a complication of diabetes [58]. Employment of emollients can restore physiological lipid levels in the skin, improves its barrier function, reduces itch and prevents infections related to scratching [58-60].

Therapy includes normalizing glucose levels accompanied by basic skin care with emollients such as ureacontaining moisturizers, amended by topical antipruritics such as polidocanol, camphor, menthol or tannin preparations [10, 39, 61]. Emollient addition to standard diabetes therapy may reduce skin complications associated with elevated blood sugar level [39]. Although emollients represent a regular adjunct to other topical treatments for patients affected by dermatoses, studies evaluating their impact on skin improvement in DM are very few.

Despite relative effectiveness and safety of topical therapy, this method is often very difficult for patients to apply when they have a generalized itch and it also requires assistance of family members and/or caregivers.

Non-sedating antihistamines and selective serotonin reuptake inhibitor antidepressants may also reduce nondermatological itch $[62,63]$. However, there is a lack of the data assessing their usage in itch of patients with DM. The European Guideline on Chronic Pruritus [10] recommends from among systemic therapies the usage of corticosteroids with caution in patients with DM as they may cause hyperglycaemia.

\section{Conflict of interest}

The authors declare no conflict of interest.

\section{References:}

1. Cho NH, Shaw JE, Karuranga S, et al. IDF Diabetes Atlas: Global estimates of diabetes prevalence for 2017 and projections for 2045. Diabetes Res Clin Pract 2018; 138: 271-81.

2. Araszkiewicz A, Bandurska-Stankiewicz E, Budzyński A, et al. 2019 Guidelines on the management of diabetic patients. A position of Diabetes Poland. Clin Diabetol 2019; 8: 1-95.

3. Bruno G, Runzo C, Cavallo-Perin P, et al. Incidence of type 1 and type 2 diabetes in adults aged 30-49 years: the population-based registry in the province of Turin, Italy. Diabetes Care 2005; 28: 2613-9.

4. Ezzati M, Hoorn SV, Lopez AD, et al. Comparative Quantification of Mortality and Burden of Disease Attributable to Selected Risk Factors. The International Bank for Reconstruction and Development/The World Bank 2006.

5. Singh N, Armstrong DG, Lipsky BA. Preventing foot ulcers in patients with diabetes. JAMA 2005; 293: 217.
6. Yancovitz M, Johnson H, Wang N, Pomeranz MK. Perforating collagenosis. Dermatol Online J 2008; 14: 14.

7. Wagner G, Sachse MM. Acquired reactive perforating dermatosis. J Der Dtsch Dermatologischen Gesellschaft 2013; 11: 723-9.

8. Ständer S, Streit M, Darsow U, et al. Diagnostic and therapeutic procedures in chronic pruritus. I Dtsch Dermatol Ges 2006; 4: 350-70.

9. Steinhoff M, Bienenstock J, Schmelz M, et al. Neurophysiological, neuroimmunological, and neuroendocrine basis of pruritus. J Invest Dermatol 2006; 126: 1705-18.

10. Weisshaar E, Szepietowski J, Darsow U, et al. European guideline on chronic pruritus. Acta Derm Venereol 2012; 92: 563-81.

11. Szepietowski JC, Reich A. Świąd. Patomechanizm, klinika, leczenie. Termedia, Poznań 2010.

12. Ständer S, Weisshaar E, Mettang T, et al. Clinical classification of itch: a position paper of the international forum for the study of itch. Acta Derm Venereol 2007; 87: 291-4.

13. Alizadeh N, Mirpour SH, Golmohamadi R, et al. Chronic generalized pruritus without primary skin lesions: a longitudinal prospective observational study. Int J Dermatol 2019; 58: 273-8.

14. Steinhoff M, Schmelz M, Szabó IL, Oaklander AL. Clinical presentation, management, and pathophysiology of neuropathic itch. Lancet Neurol 2018; 17: 709-20.

15. Tseng HW, Ger LP, Liang CK, et al. High prevalence of cutaneous manifestations in the elderly with diabetes mellitus: an institution-based cross-sectional study in Taiwan. J Eur Acad Dermatol Venereol 2015; 29: 1631-5.

16. Yamaoka H, Sasaki H, Yamasaki H, et al. Truncal pruritus of unknown origin may be a symptom of diabetic polyneuropathy. Diabetes Care 2010; 33: 150-5.

17. Lyell A. The itching patient. Scott Med J 1972; 17: 334-47.

18. Farshchian M, Farshchian M, Fereydoonnejad M, et al. Cutaneous manifestations of diabetes mellitus: a case series. Cutis 2010; 86: 31-5.

19. Moher D, Liberati A, Tetzlaff J, Altman DG; PRISMA Group. Preferred reporting items for systematic reviews and meta-analyses: the PRISMA statement. PLoS Med 2009; 6: e1000097.

20. Kantor GR, Lookingbill DP. Generalized pruritus and systemic disease. J Am Acad Dermatol 1983; 9: 375-82.

21. Neilly JB, Martin A, Simpson N, MacCuish AC. Pruritus in diabetes mellitus: investigation of prevalence and correlation with diabetes control. Diabetes Care 1986; 9: 273-5.

22. Ko MJ, Chiu HC, Jee SH, et al. Postprandial blood glucose is associated with generalized pruritus in patients with type 2 diabetes. Eur J Dermatol 2013; 23: 688-93.

23. Greenwood AM. A study of the skin in five hundred cases of diabetes. J Am Med Assoc 1927; 89: 774-7.

24. Scribner M. Diabetes and pruritus of the scalp. J Am Med Assoc 1977; 237: 1559.

25. Valdes-Rodriguez R, Mollanazar N, González-Muro J, et al. Itch prevalence and characteristics in a hispanic geriatric population: a comprehensive study using a standardized itch questionnaire. Acta Derm Venereol 2015; 95: 417-21.

26. Mishra VN, Mansharmani GG. Diabetes mellitus in geriatric females. J Indian Med Assoc 1989; 87: 138-9.

27. Reszke R, Pełka D, Walasek A, et al. Skin disorders in elderly subjects. Int J Dermatol 2015; 54: e332-8.

28. Hillson RM, Hockaday TD, Newton DJ, Pim B. Delayed diagnosis of non-insulin-dependent diabetes is associated with 
greater metabolic and clinical abnormality. Diabet Med 1985; 2: 383-6.

29. Nigam PK, Pande S. Pattern of dermatoses in diabetics. Indian J Dermatol Venereol Leprol 2003; 69: 83-5.

30.Sasmaz S, Buyukbese M, Cetinkaya A, et al. The prevalence of skin disorders in type-2 diabetic patients. Internet J Dermatolol 2004; 3.

31. Raina S, Kaushal S, Mahajan V, et al. Pattern of cutaneous manifestations in diabetes mellitus. Indian J Dermatol 2010; 55: 39-41.

32. Ghosh K, Das K, Ghosh S, et al. Prevalence of skin changes in diabetes mellitus and its correlation with internal diseases: a single center observational study. Indian J Dermatol 2015; 60: 465-9.

33. Ahmed K, Muhammad Z, Qayum I. Prevalence of cutaneous manifestations of diabetes mellitus. J Ayub Med Coll Abbottabad 2009; 21: 76-9.

34. ElFangary M, ElNemisy N, Sanad E, Sorour N. Skin manifestations in Egyptian diabetic patients: a case series study. Egypt J Dermatology Venerol 2013; 33: 56-62.

35. Al-Mutairi N, Zaki A, Sharma AK, Al-Sheltawi M. Cutaneous manifestations of diabetes mellitus. Med Princ Pract 2006; 15: 427-30.

36. Drivsholm T, de Fine Olivarius N, Nielsen ABS, Siersma V. Symptoms, signs and complications in newly diagnosed type 2 diabetic patients, and their relationship to glycaemia, blood pressure and weight. Diabetologia 2005; 48: 210-4.

37. Wahid Z, Kanjee A. Cutaneous manifestations of diabetes mellitus. J Pak Med Assoc 1998; 48: 304-5.

38. Yosipovitch G, Hodak E, Vardi P, et al. The prevalence of cutaneous manifestations in IDDM patients and their association with diabetes risk factors and microvascular complications. Diabetes Care 1998; 21: 506-9.

39. Seité S, Khemis A, Rougier A, Ortonne J. Importance of treatment of skin xerosis in diabetes. J Eur Acad Dermatology Venereol 2011; 25: 607-9.

40. Franzén LE, Roberg K. Impaired connective tissue repair in streptozotocin-induced diabetes shows ultrastructural signs of impaired contraction. J Surg Res 1995; 58: 407-14.

41. Brown RL, Breeden MP, Greenhalgh DG. PDGF and TGF-alpha act synergistically to improve wound healing in the genetically diabetic mouse. J Surg Res 1994; 56: 562-70.

42. Bitar MS. Glucocorticoid dynamics and impaired wound healing in diabetes mellitus. Am J Pathol 1998; 152: 547-54.

43. Kennedy L, Baynes JW. Non-enzymatic glycosylation and the chronic complications of diabetes: an overview. Diabetologia 1984; 26: 93-8.

44. Sternberg M, Cohen-Forterre L, Peyroux J. Connective tissue in diabetes mellitus: biochemical alterations of the intercellular matrix with special reference to proteoglycans, collagens and basement membranes. Diabete Metab 1985; 11: 27-50.

45. Tsao MC, Walthall BJ, Ham RG. Clonal growth of normal human epidermal keratinocytes in a defined medium. J Cell Physiol 1982; 110: 219-29.

46. Ando Y, Jensen PJ. Epidermal growth factor and insulin-like growth factor I enhance keratinocyte migration. J Invest Dermatol 1993; 100: 633-9.

47. Benoliel AM, Kahn-Peries B, Imbert J, Verrando P. Insulin stimulates haptotactic migration of human epidermal keratinocytes through activation of NF-kappa B transcription factor. J Cell Sci 1997; 110: 2089-97.

48. Wertheimer E, Trebicz M, Eldar T, et al. Differential roles of insulin receptor and insulin-like growth factor-1 receptor in differentiation of murine skin keratinocytes. J Invest Dermatol 2000; 115: 24-9.

49. Sakai S, Kikuchi K, Satoh J, et al. Functional properties of the stratum corneum in patients with diabetes mellitus: similarities to senile xerosis. Br J Dermatol 2005; 153: 319-23.

50.Sakai S, Endo Y, Ozawa N, et al. Characteristics of the epidermis and stratum corneum of hairless mice with experimentally induced diabetes mellitus. J Invest Dermatol 2003; 120: 79-85.

51. Seirafi H, Farsinejad K, Firooz A, et al. Biophysical characteristics of skin in diabetes: a controlled study. J Eur Acad Dermatology Venereol 2009; 23: 146-9.

52. Young MJ, Boulton AJM, Macleod AF, et al. A multicentre study of the prevalence of diabetic peripheral neuropathy in the United Kingdom hospital clinic population. Diabetologia 1993; 36: 150-4.

53. Sun PC, Chen CS, Kuo CD, et al. Impaired microvascular flow motion in subclinical diabetic feet with sudomotor dysfunction. Microvasc Res 2012; 83: 243-8.

54. Afsar B, Elsurer Afsar R. HbAlc is related with uremic pruritus in diabetic and nondiabetic hemodialysis patients. Ren Fail 2012; 34: 1264-9.

55. Narbutt J, Bednarski IA, Lesiak A. The effect of an emollient with benfothiamine and Biolin prebiotic on the improvement of epidermal skin function. Adv Dermatol Allergol 2016; 33: 224-31.

56. Ibrahim IM, Elsaie ML, Almohsen AM, Mohey-Eddin MH. Effectiveness of topical clove oil on symptomatic treatment of chronic pruritus. J Cosmet Dermatol 2017; 16: 508-11.

57. Ryeal Hahm J, Kim BJ, Kim KW. Clinical experience with thioctacid (thioctic acid) in the treatment of distal symmetric polyneuropathy in Korean diabetic patients. J Diabetes Complications 2004; 18: 79-85.

58. Proksch E. The role of emollients in the management of diseases with chronic dry skin. Skin Pharmacol Physiol 2008; 21: $75-80$.

59. Loden M. Role of topical emollients and moisturizers in the treatment of dry skin barrier disorders. Am J Clin Dermatol 2003; 4: 771-88.

60.Loden M. The clinical benefit of moisturizers. J Eur Acad Dermatology Venereol 2005; 19: 672-88.

61. Pham HT, Exelbert L, Segal-Owens AC, Veves A. A prospective, randomized, controlled double-blind study of a moisturizer for xerosis of the feet in patients with diabetes. Ostomy Wound Manage 2002; 48: 30-6.

62. O'Donoghue M, Tharp MD. Antihistamines and their role as antipruritics. Dermatol Ther 2005; 18: 333-40.

63.Zylicz Z, Krajnik M, van Sorge AA, Costantini M. Paroxetine in the treatment of severe non-dermatological pruritus: a randomized, controlled trial. J Pain Symptom Manage 2003; 26: 1105-12. 